PSYCHOMETRIKA--VOL. 31 , No. 2

JUNE, 1966

\title{
DERIVATION OF LEARNING PROCESS STATISTICS FOR A GENERAL MARKOV MODEL*
}

\author{
Harley A. Bernbach $\dagger$ \\ UNIVERSITY OF MICHIGAN
}

\begin{abstract}
A Markov learning model may be stated in the form of a transition matrix, starting vector, and response probability vector. Utilizing these and some general properties of absorbing Markov chains, general expressions are derived for several statistics of the learning process which can be applied to any model of this form. Included are derivations for the mean learning curve, number of total errors, trial numbers of the first success and the last error, and the number of error runs. As an illustration, all derivations are worked out for the simple two-state one-element model.
\end{abstract}

\section{Introduction}

Situations in which learning can be characterized by an increase in the probability of success which approaches an asymptote at a probability of unity have been described by models based on absorbing Markov chains $[1,2,3,4,5,8]$. Bower [3], presenting the simplest such model (the two-state one-element model), noted the great mathematical tractability of the system, and derived expressions for a large number of properties of the learning process which could be used to test the theory. While these derivations remain possible in the case of more complicated models [e.g., 1, 2, 8], they become considerably more unwieldy as the number of states increases. This is mainly due to the nature of the derivations, which have typically been based on difference equations drawn from the axioms of the model.

In this paper, derivations of several learning process statistics will be presented for a general Markov model, using principally the theory of finite Markov chains as presented by Kemeny and Snell [6]. The power of Markov chain mathematics will be seen to give tractability to even the most complicated Markov model. In fact, it is possible to write a general computer program directly from the equations given here (which are in vector notation) and use the computer to generate predictions for any particular model and set of parameter values. 1231-01.

*This work was partially supported by Public Health Service Grant NIH 1 T1 GM

$\dagger$ Appreciation is expressed to David Birch for his continuing advice and support during the course of this work, and to Frank Goode for his helpful criticism of the manuscript and notation. The author is now at Cornell University. 
In general, a Markov model consists of a transition matrix, starting state probability vector, and response probability vector. The matrix operates on the state probability vector to describe the learning effect of a trial, and the response probabilities on any trial are given by the product of the response vector and the state probability vector for that trial. Further, in the type of model to be discussed here, the matrix has at least one absorbing state, and the probability of success in any absorbing state is 1 . The derivations which follow are applicable to any model that has this form.

\section{Notation}

We frst introduce the notation which will be used in the derivations.

\section{Transition Matrix}

$T=$ transition matrix whose entry $t_{i j}$ is the conditional probability that the process is in state $j$ on trial $k+1$ given that it was in state $i$ on trial $k$. The states and probabilities must be defined in such a way that each response probability is either 1 or 0 . The matrix is written in canonical form as follows:

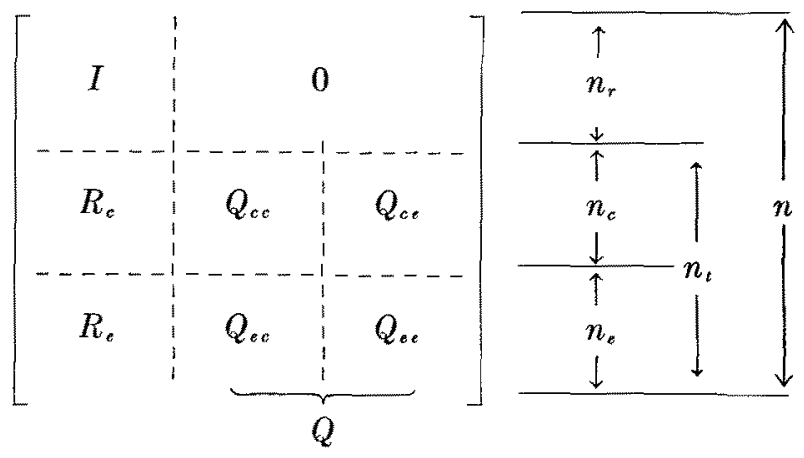

$n=$ number of states in $T$.

$n_{\mathrm{s}}=$ number of transient states (nonabsorbing states).

$n_{c}=$ number of transient correct states, i.e., transient states in which the probability of a correct response is 1 .

$n_{0}=$ number of error states.

$Q=n_{t} \times n_{t}$ submatrix of $T$ consisting of transient states only.

$Q_{c c}=n_{c} \times n_{c}$ submatrix of $T$ representing transition probabilities from the transient correct to the transient correct states.

$Q_{c e}=n_{c} \times n_{e}$ submatrix of $T$ representing transition probabilities from transient correct to error states.

$Q_{e c}=n_{\mathrm{e}} \times n_{c}$ submatrix of $T$ representing transition probabilities from error to transient correct states. 
$Q_{. .}=n_{\bullet} \times n_{e}$ submatrix of $T$ representing transition probabilities from error to error states.

$R_{c}=n_{c} \times n_{r}$ submatrix of $T$ representing transition probabilities from transient correct to absorbing states.

$R_{e}=n_{e} \times n_{r}$ submatrix of $T$ representing transition probabilities from error to absorbing states.

$N=$ fundamental matrix of $T . \quad N=(I-Q)^{-1}$.

$N_{\text {e. }}=\left(I-Q_{\text {ce }}\right)^{-1}$.

$N_{c c}=\left(I-Q_{c c}\right)^{-1}$.

State and Response Vectors

$W_{k}=1 \times n$ vector of probabilities of being in each state on trial $k$.

$W_{k, t}=1 \times n_{t}$ vector of probabilities of each transient state on trial $k$.

$W_{k, \bullet}=1 \times n_{e}$ vector of probabilities of each error state on trial $k$.

$W_{k, c}=1 \times n_{c}$ vector of probabilities of each transient correct state on trial $k$.

$P=n \times 1$ vector of correct response probabilities.

$P^{*}=n \times 1$ vector of error probabilities.

$P_{t}^{*}=n_{t} \times 1$ vector of error probabilities for transient states.

$p_{i}^{*} \quad=i$ th entry of $P_{t}^{*}$, i.e., probability of an error when process is in state $i$.

Random Variables

$A_{k}=$ response random variable, which takes on the value 0 if a correct response is made on trial $k$ and the value 1 if an error is made on trial $k$.

$X=$ total number of errors before absorption.

$Y_{i}=$ number of times process is in state $i$ before absorption.

$G=$ number of errors before first correct response.

$F=$ trial number of first correct response.

$L=$ trial number of last error.

$S_{k}=$ random variable which takes on the value 1 if a correct response is made on trial $k-1$ and an error on trial $k$, and the value 0 otherwise.

$R=$ number of error runs before absorption.

Other Notation

$Y=1 \times n_{i}$ vector entries $E\left(Y_{i}\right)$, expected number of times the process is in state $i$ before absorption.

$B=n_{c} \times 1$ vector whose $i$ th entry is the probability that there will be no errors subsequent to a response in transient correct state $i$.

$C=$ column vector with all entries 1 , which serves to sum the entries of any vector which multiplies it.

$E$. = matrix with each entry 1 or 0 , constructed so as to leave only the entries for the error states in a vector which multiplies it.

$E_{c}=$ matrix which leaves only the entries for transient correct states in a vector which multiplies it. 


\section{Derivations}

\section{Learning Curve}

In a Markov model, the transition matrix relates the state probabilities on successive trials by the formula

$$
W_{k+1}=W_{k} T
$$

By substitution, we obtain

$$
W_{k}=W_{1} T^{i-1}
$$

where $W_{1}$ is the starting vector which is given by the model. The probabilities of a correct response is simply

$$
\operatorname{Pr}\left(A_{k}=0\right)=W_{k} P \text {. }
$$

Number of Total Errors

Since an error is made if and only if the process is in an error state, the number of total errors is the same as the total number of times that the process is in an error state before absorption. Thus, summing over the set of error states, we have

$$
X=\sum Y_{i}
$$

Taking the expectation, we get

$$
E(X)=\sum E\left(Y_{i}\right)
$$

which may be written in vector notation as

$$
E(X)=Y E_{\text {. }} \text {. }
$$

As shown by Kemeny and Snell ([6], p. 46), the entry $n_{i j}$ of the fundamental matrix $N$ gives the mean number of times that an item starting in state $i$ is in state $j$ before absorption. Thus, we may write

$$
Y=W_{1, t} N
$$

and substituting into (3),

$$
E(X)=W_{1, t} N E_{\bullet}
$$

\section{Trial Number of the First Success}

To determine the mean number of errors before the first correct response, we make all correct states of $T$ absorbing, so that the process will be absorbed as soon as a correct response occurs. There will be no contribution to this mean when the process starts in a correct state, and the mean time before absorption starting in an error state can be computed directly from $Q_{\text {.s. }}$. 
Since the mean time before absorption is the sum of the mean times in each transient state, we have

$$
E(G)=W_{1, e} N_{e,} C .
$$

The mean trial number of the first success is simply

$$
E(F)=1+W_{1, e} N_{\odot e} C .
$$

Trial Number of the Last Error

In order for the last error to occur on trial $k$, it is necessary that the process be in an error state on trial $k$, and then either move to a transient correct state on trial $k+1$ and be followed by no subsequent errors or move to an absorbing state on trial $k+1$. Thus, we have

$$
\operatorname{Pr}(L=k)=W_{k, e} Q_{e c} B+W_{k, e} R_{e} C .
$$

To find $B$, we make all the error states of $T$ absorbing, so that the $i$ th entry of $B$ will be the probability that an item starting in state $i$ will be absorbed in a correct state (one of the absorbing states of the original matrix) rather than in an error state. The matrix can be rearranged to the canonical form

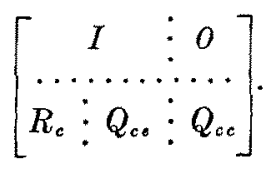

Again using a result by Kemeny and Snell ([6], p. 52), have

$$
B=N_{c c} R_{c} C \text {, }
$$

and substituting into (6)

$$
\operatorname{Pr}(L=k)=W_{k, e} Q_{\bullet c} N_{c c} R_{c} C+W_{k, e} R_{e} C .
$$

The expected trial of the last error is given by

$$
\begin{aligned}
E(L) & =\sum_{k=1}^{\infty}[k \operatorname{Pr}(L=k)] \\
& =\left(\sum_{k=1}^{\infty} k W_{k, e}\right) Q_{c c} N_{c c} R_{c} C+\left(\sum_{k=1}^{\infty} k W_{k, .}\right) R_{s} C .
\end{aligned}
$$

The sum is taken as follows. Note that the cell entries of the powered submatrix $Q^{k}$ are identical to the corresponding entries of the powered complete matrix $T^{k}$. This is a result of the property of absorbing Markov chains that it is not possible to return to the set of transient states once an absorbing state is reached. Since there is no absorbing error state, $W_{k, \text { s }}$ may be written in terms of the submatrix $Q$ as

$$
W_{k, \varepsilon}=W_{1, \imath} Q^{k-1} E_{\text {e }},
$$


and by substitution,

$$
\sum_{k=1}^{\infty} k W_{k, e}=W_{1, \perp}\left(\sum_{k=1}^{\infty} k Q^{k-1}\right) E_{.} .
$$

But, since the powers of $Q$ tend to $0([6]$, p. 44$)$, we can write

$$
\sum_{k=1}^{\infty} k Q^{k-1}=\left[(I-Q)^{-1}\right]^{2}=N^{2},
$$

and

$$
\sum_{k=1}^{\infty} k W_{k, \bullet}=W_{1, t} N^{2} E_{e} .
$$

The final expression for the mean trial number of the last error is

$$
E(L)=W_{1, t} N^{2} E_{e} Q_{e c} N_{c c} R_{c} C+W_{1, t} N^{2} E_{e} R_{e} C .
$$

Mean Number of Error Runs

An error run starts whenever there is an error following a success, or an error on the first trial. Therefore, a statistic which counts success-error sequences will serve to count error runs. The probability that such a sequence starts on trial $k$ is given by

$$
\operatorname{Pr}\left(S_{k}=1\right)= \begin{cases}W_{1} P^{*}, & \text { if } k=1, \\ W_{n, c} Q_{c o} C, \quad n=k-1, & \text { if } k>1 .\end{cases}
$$

The mean number of error runs is given by

$$
\begin{aligned}
E(R) & =\sum_{k=1}^{\infty} \operatorname{Pr}\left(S_{k}=1\right) \\
& =W_{1} P^{*}+\sum_{k=1}^{\infty} W_{k, c} Q_{c s} C .
\end{aligned}
$$

The summation is taken by the same procedure used to derive (10). Note that

$$
W_{k, c}=W_{1, \ell} Q^{k-1} E_{c} .
$$

The sum can be written

$$
\begin{aligned}
\sum_{k=1}^{\infty} W_{k, \bullet} & =W_{1, t} \sum_{k=1}^{\infty} Q^{k-1} E_{c}, \\
& =W_{1, t} N E_{c},
\end{aligned}
$$

and by substitution,

$$
E(R)=W_{1} P^{*}+W_{1, t} N E_{c} Q_{c e} C .
$$


Application to the One-Element Model

As an illustration of the use of the expressions developed above, the derivations will be applied to the one-element model, and algebraic expressions will be developed which compare with those derived by Bower [3].

This model can be written as

$$
\begin{aligned}
& T=\left[\begin{array}{ccc}
1 & 0 & 0 \\
c & (1-c) / n & (1-c)(1-1 / n) \\
c & (1-c) / n & (1-c)(1-1 / n)
\end{array}\right], \\
& W_{1}=\left[\begin{array}{lll}
0 & 1 / n & 1-1 / n
\end{array}\right], \\
& P=\left[\begin{array}{l}
1 \\
1 \\
0
\end{array}\right]
\end{aligned}
$$

\section{Learning Curve}

The powers of the matrix $T$ are given by

$$
T^{k}=\left[\begin{array}{ccc}
1 & 0 & 0 \\
1-(1-c)^{k} & (1-c)^{k} / n & (1-c)^{k}(1-1 / n) \\
1-(1-c)^{k} & (1-c)^{k} / n & (1-c)^{k}(1-1 / n)
\end{array}\right],
$$

so we have

$$
\begin{aligned}
W_{k} & =\left[1-(1-c)^{k-1}(1-c)^{k-1} / n \quad(1-c)^{k-1}(1-1 / n)\right], \\
\operatorname{Pr}\left(A_{k}=0\right) & =1-(1-c)^{k-1}+(1-c)^{k-1} / n \\
& =1-(1-1 / n)(1-c)^{k-1} .
\end{aligned}
$$

Number of Total Errors

The fundamental matrix is given by

$$
\begin{aligned}
N & =(I-Q)^{-1} \\
& =\left[\begin{array}{rr}
1-(1-c) / n & -(1-c)(1-1 / n) \\
-(1-c) / n & 1-(1-c)(1-1 / n)
\end{array}\right]^{-1}, \\
& =\frac{1}{c}\left[\begin{array}{cc}
1-(1-c)(1-1 / n) & (1-c)(1-1 / n) \\
(1-c) / n & 1-(1-c) / n
\end{array}\right] .
\end{aligned}
$$

Substituting into (4), we obtain

$$
E(X)=\frac{1-1 / n}{c} .
$$


Trial Number of First Success we have

The submatrix $Q_{\text {oe }}$ contains the single entry $(1-c)(1-1 / n)$. Thus,

$$
N_{\mathrm{ce}}=\frac{1}{1-(1-c)(1-1 / n)},
$$

and, from (5),

$$
E(F)=1+\frac{1-1 / n}{1-(1-c)(1-1 / n)} .
$$

Trial Number of Last Error

We first find $B$, as follows:

$$
N_{c c}=\frac{1}{1-(1-c) / n}, \quad R_{c}=c, \quad C=1 .
$$

Now, from (7),

$$
B=\frac{c}{1-(1-c) / n} \text {. }
$$

Further,

$$
W_{k, e}=(1-c)^{k-1}(1-1 / n), \quad Q_{e c}=(1-c) / n, \quad R_{e}=c .
$$

Substituting into (6),

$$
\operatorname{Pr}(L=k)=\frac{c(1-1 / n)(1-c)^{k-1}}{1-(1-c) / n} .
$$

To find the mean trial number of the last error, we first find the sum $\sum k W_{k, \text { f }}$ from (10):

$$
W_{1, t}=\left[\begin{array}{ll}
1 / n & 1-1 / n
\end{array}\right], \quad E_{\varepsilon}=\left[\begin{array}{l}
0 \\
1
\end{array}\right]
$$

and using $N$ from (16),

$$
\sum k W_{k, 0}=\frac{1-1 / n}{-c^{2}} .
$$

From (9) it follows that the mean trial of the last error is

$$
E(L)=\frac{1-1 / n}{c[1-(1-c) / n]} .
$$

Mean Number of Error Runs

The sum $\sum W_{k, c}$ is found in the same manner as (21), except that we use

$$
E_{c}=\left[\begin{array}{l}
1 \\
0
\end{array}\right]
$$


We have

$$
\sum W_{k, c}=\frac{1 / n}{c}
$$

Now, since

$$
Q_{c e}=(1-c)(1-1 / n),
$$

it follows from (13) that

$$
E(R)=\frac{1-1 / n}{c}[1-(1-c)(1-1 / n)] .
$$

\section{Discussion}

While it is a simple matter in the case of the one-element model to develop algebraic expressions from the vector equations derived in this paper, this becomes far more difficult when the matrix $T$ contains more than two states. The derivations are not intended, however, to provide an alternate route to the same expressions that might be derived from difference equations. Instead, it is expected that the vector equations will be used in their present form, i.e., first numerical values will be substituted for the entries of the probability vectors and matrix which define the model, and then the statistics of the learning process will be calculated from these. As mentioned previously, it is possible to write a computer program directly from the vector equations to perform these operations.

The major advantage of this approach is that it makes mathematically tractable even the most complicated Markov model, so long as it can be written in the form of a transition matrix and pair of probability vectors. This is useful in the case of a model which permits considerable movement between transient states prior to absorption, such as [2]. More important, however, this approach makes it possible to develop systems with many states, such as might be suggested by multiprocess learning theories [e.g., 7], without losing the necessary ability to derive testable consequences of the theory.

\section{REFERENCES}

[1] Atkinson, R. C. and Crothers, E. J. A comparison of paired-associate learning models having different acquisition and retention axioms. $J$. math. Psychol., 1964, 1, 285-315.

[2] Bernbach, H. A. A forgetting model for paired-associate learning. J. math. Psychol., 1965, 2, 128-144.

[3] Bower, G. H. Application of a model to paired-associate learning. Psychometrika, 1961, 26, 255-280.

[4] Bower, G. H. and Trabasso, T. Concept identification. In R. C. Atkinson (Ed.), Studies in mathematical psychology. Stanford: Stanford Univ. Press, 1964.

[5] Estes, W. K. Component and pattern models with Markovian interpretations. In 
R. R. Bush and W. K. Estes (Eds.), Studies in mathematical learning theory. Stanford: Stanford Univ. Press, 1959.

[6] Kemeny, J. G. and Snell, J. L. Finite Markov chains. Princeton, N. J.: D. Van Nostrand, 1959.

[7] MeGuire, W. J. A multiprocess model for paired-associate learning. J. exp. Psychol., 1961, 62, 335-347.

[8] Theios, J. Simple conditioning as two-stage all-or-none learning. Psychol. Rev., 1963, $70,403-417$.

Manuscript received 9/17/64

Revised manuscript received 1/25/65 